\section{Sindbis vectors: illuminating the path to ovarian cancer therapy}

K-W Peng and E Galanis

Gene Therapy (2005) 12, 381-382. doi:10.1038/sj.gt.3302452

Published online 23 December 2004

With an estimated 25500 new cases and 16000 deaths in 2004, ovarian cancer is the fourth biggest cancer killer among American women. ${ }^{1}$ Since early stage disease is often asymptomatic, $70 \%$ of patients present with advanced disease at the time of diagnosis. While $70 \%$ of patients relapse and available salvage therapies are not curative, ${ }^{2}$ diagnostic methods for early detection and novel therapeutic approaches are urgently needed. In the September issue of Cancer $R e$ search, ${ }^{3}$ Tseng et $a l^{4}$ have now extended their previous observations on the potential use of Sindbis vectors as probes for in vivo localization of ovarian cancer and as novel antitumor agents.

Recurrent ovarian cancer is a good candidate for intraperitoneal gene transfer/virotherapy approaches as it remains confined in the peritoneal cavity in $85 \%$ of patients with advanced disease. Several such gene transfer and virotherapy trials have now been completed. ${ }^{5}$ From these clinical trials, it has become obvious, that one of the major hindrances in optimizing gene transfer strategies, pertains to the difficulty in monitoring gene expression and viral replication in vivo. This frequently makes selection of the phase II dose empiric rather than based on comprehensive assessment of biologic activity. Furthermore, it has been difficult to localize the therapeutic gene in tumor cells in vivo.

Sindbis viruses have already been explored as gene delivery vehicles. ${ }^{6}$ Sindbis is the prototype member of the Alphavirus genus, consisting of 30 blood-borne human or animal pathogens. They are replication defective and the infected cell produces no progeny viruses. However, the packaged vector genomes are selfreplicating and can amplify in the host cell to drive high levels of transgene expression. ${ }^{9}$ Also, replica- tion of viral RNA and expression of nonstructural proteins induce apoptosis and cell death within 1 to 3 days after infection, ${ }^{7}$ making Sindbis very attractive as a cytotoxic virus for cancer therapy. In addition, because Sindbis is a blood-borne virus, Sindbis vectors are suitable for systemic administration, which represents an advantage in systemic therapy of metastatic disease. ${ }^{8}$

Tseng et $a l^{3,4}$ gave a new dimension to potential applications of the Sindbis vectors by using them as probes to detect human and murine ovarian tumors. They administered Sindbis vectors expressing the firefly luciferase gene (Sindbis/Fluc) to the peritoneal cavity of SCID mice bearing intraperitoneal human ES-2 clear cell ovarian cancer or C57BL/ 6 mice bearing syngenic murine MOSEC ovarian tumors. After 1 day, they gave the mice luciferin substrate and imaged the mice noninvasively. Bioluminescent signals were detected in Sindbis/Fluc-injected tumor-bearing mice. Upon explantation and close examination of the tissues, the authors determined that the bioluminescent signals originated from infected tumor cells and not murine tissues.

These experiments revealed that the Sindbis vector is tumor specific, possibly due to tumor cell overexpression of a $67 \mathrm{kDa}$ high-affinity laminin receptor (LAMR) through which viral entry occurs. LAMR is substantially upregulated in numerous human cancers, including ovarian cancer (68-87\% of the patients). Higher expression of LAMR is linked with increased invasiveness and malignant potential of different neoplasms, ${ }^{10}$ and in contrast to normal cells, the majority of the LAMR in cancer cells are not occupied by laminin. ${ }^{11}$ The authors demonstrated the impact of LAMR expression on viral infectivity by generating a stable clone of ES-2 cells expressing a small interfering RNA against the LAMR. This clone expresses $40 \%$ less LAMR and resulted in a 50\% decrease in vector infectivity. Whether the tumor specificity of Sindbis virus is solely due to a differential expression in LAMR remains to be seen as the Sindbis nsp-2 protein, which is required for replication and transcription of the viral RNAs, also plays an important role in suppressing interferon-mediated anti-viral response in infected cells. ${ }^{12}$

If the Sindbis vector only infects tumor cells expressing high LAMR levels, this could impose a limitation to the widespread use of the vector as a cancer probe because some cancers may not express sufficient levels of LAMRs, or there may be heterogeneity in receptor expression levels. In that case, cancer cells expressing 'low' levels of LAMR are less likely to be infected by the vector. Furthermore, the role of heparan sulfate in the Sindbis vector/ tumor cell interaction and its impact on selectivity needs to be further characterized. ${ }^{13}$

Since 1989, more than 650 cancer gene therapy clinical trials have been initiated (Journal of Gene Medicine website) but information on the pharmacokinetics and pharmacodynamics of transgene expression has been limited. We do not know if the lack of efficacy in some of these trials is due to poor vector performance or poor choice of transgene. Are the transgenes expressed, and if so, how high and for how long? There has been tremendous progress made in the technological aspect of noninvasive monitoring to address this concern, ${ }^{14}$ and various types of strategies have been developed, including the use of soluble marker peptides to track the profile of transgene expression. Indeed, the first recombinant measles virus approved for phase I clinical testing for ovarian cancer contains a soluble CEA solely for the purpose of monitoring the viral gene expression profile. ${ }^{15,16}$ Multimodality imaging strategies to determine sites and levels of transgene expression based on light (bioluminescence), near-infra-red and radioactivity are also being pursued. ${ }^{14}$

Tseng et al elegantly demonstrated the value of including noninvasive imaging to follow the efficiency of gene transfer and monitoring therapeutic outcome. Using ES-2 cells 
stably expressing firefly luciferase (Fluc) and Sindbis vector expressing Renilla luciferase (Rluc), they showed a very good correlation between tumor sites, vector delivery and transgene expression. As Tseng and coworkers pointed out, bioluminescent imaging is not ideal for application in humans because light is unlikely to penetrate through the deep tissues. Instead, imaging based on the decay of radionuclides as gamma rays or positrons is likely to be more practical in the clinic. Preclinical development of these imaging modalities using transgenes such as the thymidine kinase gene from herpes simplex virus-1 are very promising. ${ }^{14}$

As it pertains to antitumor efficacy, treatment of SCID mice bearing intraperitoneal ES-2/Fluc tumors with the Sindbis vectors, expressing the LacZ gene, significantly suppressed tumor growth in treated mice. Furthermore, Sindbis vectors expressing the murine IL-12 and IL15 genes significantly suppressed tumor growth as compared to mice treated with Sindbis/Lac-Z, possibly through recruitment of natural killer cells and mobilization of macrophages, although this was not demonstrated. Use of Sindbis vector in a MOSEC syngeneic ovarian cancer model demonstrated tumor-specific infection and prolongation of survival in treated animals. Despite the positive impact on survival, no animals in the treated group were cured and they all eventually succumbed. The authors hypothesized from previous data in other animal models that the antitumor activity of the Sindbis vector was due to induction of apoptosis. ${ }^{17}$ Nevertheless, the impact of anti-viral humoral and cellular immune response on treatment efficacy after repeat administration will need to be more thoroughly investigated.
In summary, in the current and previous works, ${ }^{3}$ Tseng et al highlighted two important areas for cancer research and gene therapy: (1) in vivo localization of tumor cells and (2) noninvasive monitoring of transgene expression. The novel use of Sindbis/Fluc as a probe to light up the tumors during surgery could potentially make tumor identification and debulking easier. Inclusion of noninvasive monitoring of transgene expression in clinical trials could optimize viral dosing and possibly improve the therapeutic outcome. While light-based imaging is not easily translatable into the clinic for human use, it is a very appealing modality for preclinical development and monitoring of novel gene-based therapeutics, because of its convenience, safety, lack of radioactive waste and lower cost compared to gamma or PET imaging. In addition, the potency of these intrinsically cytotoxic vectors can be enhanced via use of therapeutic transgenes such as cytokine genes or possibly in combination with other treatment modalities, thus making Sindbis a potentially promising virus for clinical translation.

Dr K-W Peng and Dr E Galanis are at the Molecular Medicine Program and Department of Oncology, Mayo Clinic, Rochester, MN 55905, USA

E-mail:peng.kah@mayo.edu

Published online 23 December 2004

1 Jemal A et al. American Cancer Society. Cancer statistics, 2004. CA Cancer J Clin 2004; 54: 8-29.

2 Stuart GC. First-line treatment regimens and the role of consolidation therapy in advanced ovarian cancer. Gynecol Oncol 2003; 90: S8-S15.

3 Tseng JC et al. Using Sindbis viral vectors for specific detection and suppression of advanced ovarian cancer in animal models. Cancer Res 2004; 64: 6684-6692.
4 Tseng JC et al. Systemic tumor targeting and killing in Sindbis viral vectors. Nat Biotechnol 2004; 22: 70-77.

5 Kirby TO, Curiel DT, Alvarez RD. Gene therapy for ovarian cancer: progress and potential. Hematol Oncol Clin N Am 2003; 17: 1021-1050.

6 Yamanaka R. Alphavirus vectors for cancer gene therapy. Int J Oncol 2004; 24: 919-923.

7 Griffin DE, Hardwick JM. Regulators of apoptosis on the road to persistent alphavirus infection. Annu Rev Microbiol 1997; 51: 565-592.

8 Turrell MJ. Horizontal and vertical transmission of viruses by insect and tick vectors. In: Monath TP (ed). The Arboviruses: Epidemiology and Ecology. CRC Press Inc.: Boca Raton, FL, 1998, pp 127-152.

9 Bredenbeek PJ et al. Sindbius virus expression vectors: packaging of RNA replicons by using defective helper RNAs. J Virol 1993; 67: 6439-6446.

10 Menard S, Tagliabue E, Colnaghi MI. The $67 \mathrm{kDa}$ laminin receptor as a prognostic factor in human cancer. Breast Cancer Res Treat 1998; 52: 137-145.

11 Liotta LA et al. The laminin receptor and basement membrane dissolution: role in tumour metastasis. Ciba Found Symp 1994; 108: 146-162.

12 Frolov I. Persistent infection and suppression of host response by alphaviruses. Arch Virol Suppl 2004; 18: 139-147.

13 Byrnes AP, Griffin DE. Binding of Sindbis virus to cell surface heparan sulfate. J Virol 1998; 72: 7349-7356.

14 Shah K et al. Molecular imaging of gene therapy for cancer. Gene Therapy 2004; 11: 1175-1187.

15 Peng KW et al. Non-invasive in vivo monitoring of trackable viruses expressing soluble marker peptides. Nat Med 2002; 8: 527-531.

16 Peng KW et al. Intraperitoneal therapy of ovarian cancer using an engineered measles virus. Cancer Res 2902; 62: 4656-4662.

17 Tseng JC et al. In vivo antitumor activity of Sindbis viral vectors. J Natl Cancer Inst 2002; 94: 1790-1802. 